\title{
GLIWICE RADIOCARBON DATES XII
}

\section{ANNA PAZDUR, MIECZYSŁAW F. PAZDUR and ANDRZEJ ZASTAWNY}

Radiocarbon Laboratory, Institute of Physics, Silesian Technical University, Krzywoustego 2 PL-44-100 Gliwice, Poland

\section{INTRODUCTION}

The following list presents results of dating archaeological samples from excavations in Africa. Most results reported in this list were obtained from 1982 to 1993 for international research projects studying the origin and early development of food-producing cultures in northeastern Africa, including the Nile basin and the Sahara. Detailed information on these projects may be found in the series of conference proceedings edited by Krzyżaniak and Kobusiewicz $(1984,1989,1993)$.

Three proportional counters (L1, L2 and L3), of 2.5, 5 and 1.5 liter volume, were used for dating (Pazdur et al. 1982). Procedures for sample pretreatment, counting, statistical analysis and age calculations were essentially as described in Pazdur et al. $(1982,1985)$. All results are reported as conventional ${ }^{14} \mathrm{C}$ dates in years before $\mathrm{AD} 1950$. Dates listed with $\delta^{13} \mathrm{C}$ values have been adjusted for isotopic fractionation by normalization to $\delta^{13} \mathrm{C}=-25 \%$. Sample descriptions are essentially based on information provided by submitters.

\section{ACKNOWLEDGMENTS}

This contribution is part of research supported by grant PB 740/6/91 from the State Committee for Scientific Research.

\section{Paleolithic}

\section{Wadi Kubbaniya Series}

Charcoal from excavations of Upper Paleolithic sites of Kubbaniyan culture in Wadi Kubbaniya $\left(33^{\circ} \mathrm{N}, 24^{\prime} \mathrm{E}\right)$, Western Desert, Upper Egypt, consisting of cultural layers in dune sands (Wendorf, Schild and Close 1980, 1987). Site E-78-3 is located ca. $25 \mathrm{~km} \mathrm{~N}$ of Assuan, sites E-78-4 and E-78$5 \mathrm{ca} .15 \mathrm{~km} \mathrm{~N}$ of Assuan, at elevations of $c a .100 \mathrm{~m}$ asl. Samples from E-78-3 collected 1982 and 1983 by Angela Close, from E-78-4 collected February 1983 by Hanna Wieckowska, from E-78-5 collected 1983 by Michał Kobusiewicz. Submitted 1982 and 1983 by Romuald Schild, Institute of History of Material Culture, Polish Academy of Sciences, Warsaw.

Gd-1522. Wadi Kubbaniya E-78-3/20

$18,500 \pm 220$

From Level 20, Cut I/82, depth $150-200 \mathrm{~cm}$.

Gd-1520. Wadi Kubbaniya E-78-3/20/21

$18,110 \pm 160$

From Level 20/21, Cut I/82, depth 150-200 cm.

Gd-2091. Wadi Kubbaniya E-78-3/23

$18,140 \pm 400$

From Level 23, Grid G-21-24, depth 150-200 cm.

Gd-1610. Wadi Kubbaniya E-78-3/24

$18,470 \pm 180$

From Level 24, Grid G-21-24, depth 50-150 cm.

Gd-2092. Wadi Kubbaniya E-78-3/over 24

$18,080 \pm 350$

Dispersed in white sand overlying cultural level 24 , Grid G-21-24, depth $20-50 \mathrm{~cm}$. 
Gd-1611. Wadi Kubbaniya E-78-4/c

From Level c, depth 100-150 cm.

$17,800 \pm 170$

Gd-1612. Wadi Kubbaniya E-78-4/e

$17,640 \pm 140$

From Level e, depth $130-180 \mathrm{~cm}$.

Gd-2093. Wadi Kubbaniya E-78-4/f

From Level f, depth $150-200 \mathrm{~cm}$.

$17,620 \pm 340$

Gd-2094. Wadi Kubbaniya E-78-5/f

From Level f, depth 150-200 cm.

$15,830 \pm 220$

Comment (M.F.P.): Bluszcz and Pazdur $(1986,1987)$ discuss in detail ${ }^{14} \mathrm{C}$ and $\mathrm{TL}$ dates obtained on materials from this site. Other conventional ${ }^{14} \mathrm{C}$ dates are presented by Haas (1987), and AMS ${ }^{14} \mathrm{C}$ dates by Donahue et al. (1987); Hietala (1987) analyzes and interprets all available ${ }^{14} \mathrm{C}$ dates.

\section{NEOLITHIC}

\section{Nabta Series}

Charcoal from excavations of Early Neolithic settlement on the shore of a seasonal lake, Nabta Playa $\left(22^{\circ} 32^{\prime} \mathrm{N}, 30^{\circ} 42^{\prime} \mathrm{E}\right)$, Western Desert, Egypt, elevation $200 \mathrm{~m}$ asl. Collected 1990, 1991 and 1992 and submitted by R. Schild. Results of early excavations undertaken in 1975-1977 are discussed by Wendorf and Schild (1980) and Schild and Wendorf (1984); results of archaeobotanical studies are reported by Wendorf et al. (1992); Hedges et al. (1993) list dates recently obtained at the Oxford AMS facility.

Gd-6260. Nabta E-75-6\#1/90

Pit I/90, X/12, the lowest part of the pit.

$8260 \pm 100$

Comment (M.F.P.): The date obtained by the Oxford AMS facility on single plant macrofossils (seeds, identified by K. Wasylikowa) from Pit I/90, X-Y/12, OxA-3220, $8025 \pm 120$ BP (Hedges $e t$ al. 1993), agrees fairly well.

Gd-6257. Nabta E-75-6\#2/90

Feature 1/90, W/13.

$7770 \pm 110$

Gd-6498. Nabta E-75-6\#1/91

Feature 1/90, NEQ, X/15, base of hearth.

$7830 \pm 110$

Gd-6254. Nabta E-75-6\#3/90

Feature 1/90, Y/16.

$8550 \pm 130$

Comment (M.F.P.): Dates obtained by the Oxford AMS facility (Hedges et al. 1993) on single plant macrofossils (seeds, identified by $\mathrm{K}$. Wasylikowa) from Feature 1/90 are: W/14, OxA-3214, $8080 \pm$ 110 вP; W/14, OxA-3215, $8095 \pm 120$ вP; Y/17-17, OxA-3217, $8020 \pm 160$ BP; W/13, OxA-3218, $8050 \pm 130 \mathrm{BP}$. Our date obtained on large pieces of charred wood is too old, probably because of the "old wood effect".

Gd-4586. Nabta E-75-6\#5/90

Pit, BB/10.

$7450 \pm 120$

Gd-6258. Nabta E-75-6\#6/90

Feature 2/90, BB/12.

$7920 \pm 100$ 
Gd-6500. Nabta E-75-6\#2/91

$7910 \pm 110$

Feature $2 / 90$, single pieces of charcoal scattered at the margin of the pit.

Comment (M.F.P.): Dates agree fairly well with results obtained at the Oxford AMS facility (Hedges et al. 1993): BB/12, OxA-3222, $8060 \pm 120 \mathrm{BP}$.

Gd-4587. Nabta E-75-6\#4/90

$8600 \pm 140$

Feature 3/90, BB/17.

Comment (M.F.P.): Date obtained by the Oxford AMS facility (Hedges et al. 1993) on single plant macrofossils (Sorghum seeds, identified by K. Wasylikowa) from Feature 3/90: BB/17, OxA-3219, $7950 \pm 160 \mathrm{BP}$.

Gd-6503. Nabta E-75-6\#3/91

$7590 \pm 110$

Feature 3/90, SEQ, Square DD/19, hearth in dark brownish sand.

Gd-6506. Nabta E-75-6\#4/91

$7850 \pm 90$

Feature 1/91, Square GG-HH/22, hearth.

Gd-6507. Nabta E-75-6\#5/91

$7610 \pm 120$

Feature 1/91, NWQ, layer with charcoal ca. 3-5 cm above the floor.

Gd-5971. Nabta E-75-6\#6/91

$7960 \pm 70$

Feature $1 / 91$, Square $\mathrm{HH} / 20-21$, single pieces of charcoal from the filling of a pit.

Gd-6508. Nabta E-75-6\#7/91

$7540 \pm 110$

Pit, Square GG/10, charcoal dispersed at a depth of $95 \mathrm{~cm}$ in yellow sand.

Gd-6509. Nabta E-75-6\#8/91

$7480 \pm 110$

Base of Pit 2/90, depth $100 \mathrm{~cm}$.

Gd-6510. Nabta E-75-6\#9/91

$7330 \pm 100$

Base of Pit 1/91.

Gd-6734. Nabta E-75-6\#1/92

$6710 \pm 110$

Pit 1/90, fireplace 1 in brown sand, $\mathrm{Y} / 16$.

Gd-6733. Nabta E-75-6\#2/92

$6620 \pm 90$

From cultural layer, Trench IV/91.

Gd-6742. Nabta E-75-6\#4/92

$6750 \pm 100$

Trench $1 / 90$, from pothole in fireplace in brown sand, GG/14.

Gd-6746. Nabta E-92-8\#6/92

$3130 \pm 110$

Fireplace in cultural layer with remains of stone huts.

\section{Dakhleh Oasis Series}

Charcoal and ostrich eggshells were collected during several seasons of activities of the Dakhleh Oasis Project (DOP) expedition of the Royal Ontario Museum, directed by A. J. Mills. The Dakhleh Oasis is located in the Egyptian Sahara, with its center at $25^{\circ} 48^{\prime} \mathrm{N}$ and $29^{\circ} 05^{\prime} \mathrm{E}$. The oasis is $\mathrm{ca}$. 80 $\mathrm{km}$ long and up to $25 \mathrm{~km}$ wide, overlooked by a 400 -m-high south-facing limestone escarpment. Its floor is flat clay plain, originally lacustrine, and rises slightly northward from 100-135 $\mathrm{m}$ asl. The DOP objective is to gain a detailed understanding of the cultural and environmental history of the Dakhleh Oasis since the beginning of the Holocene. Mills (1984) presented a general outline of the 
DOP; Brookes $(1983,1989)$ presented results of geoarchaeological reconnaissance and sedimentological studies; Edwards and Hope (1989) briefly summarized results obtained in the study of Neolithic ceramics, giving a complete list of references to interim reports published by members of the DOP team; and McDonald (1990) discussed some aspects of cattle pastoralism at the site.

Samples of DOP surface subseries were collected 1990 during a surface survey for sites of the Bashendi culture at the base of a hill bearing rock art, a sandstone ridge south of the SE basin, SE corner of Dakhleh Oasis $\left(23^{\circ} 41^{\prime} \mathrm{N}, 29^{\circ} 14^{\prime} \mathrm{E}\right)$, ca. $20 \mathrm{~km} \mathrm{~S}$ of Teneida, elevation $500 \mathrm{~m}$ asl. Samples from Site 228 with artifacts of the Bashendi culture and Site 264 of the Masara culture $\left(25^{\circ} 25^{\prime} \mathrm{N}, 29^{\circ} 25^{\prime} \mathrm{E}\right)$ were collected 1989 and 1990 and submitted 1990; samples from other sites of the Bashendi culture in the SE comer of Dakhleh Oasis $\left(25^{\circ} 25^{\prime} \mathrm{N}, 29^{\circ} 22^{\prime} \mathrm{E}\right)$ were collected and submitted 1991 by Mary M. A. McDonald, Department of Archaeology, University of Calgary, Canada. Samples from a calcareous Early Holocene lake $\left(23^{\circ} 41^{\prime} \mathrm{N}, 29^{\circ} 14^{\prime} \mathrm{E}\right)$ were collected 1990 and submitted by Ian Brookes.

DOP 88 Subseries

Gd-5792. DOP 88\#1

$7200 \pm 70$

$\delta^{13} \mathrm{C}=-5.5 \%$

Ostrich eggshell from Cluster 1 on surface, Site 228, Square J6-J7, associated with potsherds of Bashendi culture, demonstrated archaeologically to be a living site with hearths and artifacts on, or slightly under, the surface, resting partly on silt or sand.

\section{Gd-4622. DOP 88\#2}

Charcoal, scattered fragments (hearth?), just under surface, Cluster 1 Site $228, \quad 6380 \pm 100$ ciated with Bashendi potsherds.

Gd-6323. DOP 88\#3

$6940 \pm 140$

Charcoal, scattered fragments from buried Feature, Level 2, Site 228, Square A6, depth 12-14 cm, associated with the Bashendi culture.

Gd-4624. DOP 88\#4

$5770 \pm 150$

Charcoal, scattered fragments within an area $30 \mathrm{~cm}$ in diameter, probably a hearth, from the surface of Cluster 2, Site 228, Square 4-5, depth 1-3 cm.

Gd-6321. DOP 88\#5

Charcoal from Stake Hollow, sandy layer below silt $\quad 7600 \pm 100$ culture, early phase.

\section{DOP 89 Subseries}

Gd-4492. DOP 89\#1

$4310 \pm 80$

Charcoal from a rockshelter of the "Sheikh Muftah" cultural unit, Site 244 (McDonald 1990).

Gd-5646. DOP 89\#2

Ostrich eggshell from surface scatter, same locality as DOP $89 \# 3$.

$5830 \pm 70$

Gd-6168. DOP 89\#3

Charcoal from Cluster $\mathrm{f}$, hearth mound, Site 254.

$\delta^{13} \mathrm{C}=0.2 \%$

Gd-6169. DOP 89\#5

$6300 \pm 110$

Charcoal from cultural layer 40 cm $\mathbf{7 3 2 0} \pm 120$

Charcal from cultural layer $40 \mathrm{~cm}$ below surface, sealed by silts, Stake Hollow, K17b, Site 228.

Gd-6170. DOP 89\#6

Charcoal from a hearth underlying the surface, Stake Hollow, J18a, Site 228.

$7360 \pm 90$ 
Gd-5654. DOP 89\#7

$6990 \pm 70$

Charcoal from a hearth underlying the surface, Stake Hollow, M18b, Site 228.

Gd-4493. DOP 89\#8

$8340 \pm 150$

Charcoal from a hearth $35 \mathrm{~cm}$ below the surface, sealed by silts, Stake Hollow, K17, Site 228. Comment: small sample, diluted with inactive $\mathrm{CO}_{2}$ for counting.

Gd-5645. DOP 89\#9

$5930 \pm 60$ $\delta^{13} \mathrm{C}=0.89 \%$

Ostrich eggshell from surface scatter around Cluster 1, hearth mound, Site 252, same locality as DOP $89 \# 10$.

Gd-4495. DOP 89\#10

$6120 \pm 250$

Charcoal from Cluster 1, hearth mound, underlying the surface. Comment: diluted with inactive $\mathrm{CO}_{2}$ for counting.

\section{DOP CVC Subseries}

Gd-5722. DOP CVC 270 s\#8

$6470 \pm 70$

Ostrich eggshell from surface scatter over an area with many hut circles.

\section{Gd-5720. DOP CVC 264 s\#7}

$8730 \pm 70$

Ostrich eggshell from surface scatter over part of the surface of the hut-circle cluster, Masara culture.

Gd-5721. DOP CVC 266 s\#6

Ostrich eggshell from surface scatter.

$7910 \pm 80$

$\delta^{13} C=-2.6 \%$

$8650 \pm 80$

Gd-5718. DOP CVC 262 s\#4

$\delta^{13} C=-2.8 \%$ o

Ostrich eggshell from surface scatter, cluster within a scatter of chipped stone, Masara culture.

\section{Gd-5717. DOP CVC 261 s\#3}

$7380 \pm 70$

$\delta^{13} \mathrm{C}=0.0 \%$

Ostrich eggshell from the surface, associated with both Masara and Bashendi artifacts, $\mathrm{N}$ part of SE basin.

Gd-5719. DOP CVC 257 s\#1

$6250 \pm 50$

$\delta^{13} C=-5.20 \%$

Ostrich eggshell from the surface, associated with artifacts of Bashendi culture, N part of SE Basin.

DOP 90 Subseries

\section{Gd-5799. DOP 90\#2}

$\mathbf{7 2 0 0} \pm \mathbf{7 0}$

$\delta^{13} \mathrm{C}=-4.48 \%$ \%

Ostrich eggshell from the surface, Stake Hollow, Site 228, Square R20, Bashendi culture, early phase. Eggshell is just now being exposed through wind action; other eggshells still remain buried within a cultural layer up to $10 \mathrm{~cm}$ thick.

Gd-6322. DOP 90\#5

$7570 \pm 110$

Charcoal around hearth, depth $10 \mathrm{~cm}$, under playa silts, Stake Hollow, Site 228, Square J16a, Level 2, Bashendi culture, sample associated with chipped stone, animal bones and ostrich eggshell. 
Gd-4623. DOP 90\#11

$6480 \pm 140$

Charcoal from the base of a cultural layer under silts, depth ca. $35 \mathrm{~cm}$, Site 228, test trench in Square L17, sample associated with chipped stone, eggshell and bone, Bashendi culture.

Gd-6318. DOP 90\#9

$8660 \pm 90$

Charcoal from a pocket of sand between sandstone slabs forming the wall of a hut circle, depth $c a$. $10 \mathrm{~cm}$, Site 264, Square I30d, Hut I29, sample associated with chipped stone, Masara culture, Early Holocene wet phase.

Gd-6320. DOP 90\#10

$8950 \pm 120$

Charcoal, layer of ash within a stone ring (hearth?) under a hut circle, depth $c a .35 \mathrm{~cm}$, Site 264, Square I29, sample associated with chipped stone, Hut I29, Masara culture, Early Holocene wet phase.

\section{DOP 91 Subseries}

Charcoal and ostrich eggshell from surface excavations of several Neolithic sites of the Bashendi culture, SE basin, SE corner of Dakhleh Oasis $\left(25^{\circ} 25^{\prime} \mathrm{N}, 29^{\circ} 22^{\prime} \mathrm{E}\right), \mathrm{ca} .20 \mathrm{~km} \mathrm{~S}$ of Teneida, Sahara Desert, SW Egypt. Collected and submitted 1991 by M. McDonald.

Gd-5993. DOP 91\#7

$5240 \pm 110$

Charcoal, scattered fragments from a layer $c a .10 \mathrm{~cm}$ below the surface of a hearth mound, Cluster i, Bashendi culture Group B, Site 254.

Gd-5983. DOP 91\#8

$5940 \pm 70$

$\delta^{13} C=-1.2 \%$ o

Ostrich eggshell, from thin surface scatter within a circle, radius $c a .50 \mathrm{~cm}$ around the hearth that yielded sample DOP 91\#7, Bashendi culture Group B, Site 254.

Gd-6529. DOP 91\#9

$5180 \pm 110$

Charcoal from hearth \#126, depth 2-10 cm, Bashendi culture Group B, Site 254.

\section{Gd-5985. DOP 91\#10}

$5630 \pm 50$

Ostrich eggshell from thin surface scatter within a circle, radius ca. $5 \mathrm{~m}$ around ${ }^{13} \mathrm{C}=0.6 \%$ \#124, Bashendi culture Group B, Site 254.

Gd-5994. DOP $91 \# 11$

$5810 \pm 80$

Charcoal, in the form of a patch $40 \mathrm{~cm} \times 25 \mathrm{~cm}$ and $4 \mathrm{~cm}$ deep, underlying the surface, Square I8d, Level 1, Site 271.

\section{Gd-6534. DOP 91\#13/\#14/\#15}

Very fine charcoal, underlying the surface; dated material was obtained by joining three subsamples collected from Squares E9a, F8a and G8b, Site 271.

\section{Gd-6538. DOP 91\#17-18}

$6360 \pm 120$

Charcoal, underlying the surface; dated material was obtained by joining two subsamples collected from the same pit on the boundary of Squares I7b and I7d, excavated separately, Site 271.

Gd-5990. DOP 91\#1

$6850 \pm 50$

Charcoal from a layer 5-10 cm below the surface of hearth mound \#21, associated with potsherds belonging to Bashendi culture Group A, Site 275. 
Gd-5981. DOP 91\#3

$7100 \pm 60$
$\delta^{13} C=-4.3 \%$

Ostrich eggshell, collected from the surface, mapping point \#17, Cluster 1, associated with potsherds of the Bashendi culture, Site 275.

\section{Gd-5984. DOP 91\#12}

$6640 \pm 70$

$\delta^{13} C=-1.2 \%$

Ostrich eggshell, collected from the surface, mapping point \#8, associated with potsherds belonging to the Bashendi culture Group A, Site 275.

Gd-5992. DOP 91\#4

$6370 \pm 70$

Charcoal from scattered fragments in a layer at depth $c a .10 \mathrm{~cm}$ below the surface of hearth mound \#44, Cluster 1, Bashendi culture Group B, Site 276.

Gd-5982. DOP 91\#6

$\mathbf{5 7 5 0} \pm \mathbf{5 0}$

$\delta^{13} C=-1.0 \%$

Ostrich eggshell from the surface around hearth \#44, Bashendi culture Group B, Site 276.

$7180 \pm 60$

Gd-5985. DOP 91\#19

$\delta^{13} C=-3.2 \%$

Ostrich eggshell from the surface excavation of an isolated stone circle (hut circle?), depth 0-20 $\mathrm{cm}$, Site 277.

Gd-6535. DOP 91\#20

$4380 \pm 120$

Very fine charcoal, underlying the surface, from an area of burning consisting of isolated patches under windblown sand filling a hut circle, Site 277.

Comment (M.M.A.McD.): There is no clear evidence for associating dated samples DOP $91 \# 19$ and DOP $91 \# 20$ with the Bashendi culture group; the date obtained on charcoal is younger than expected, probably rejuvenated by the admixture of much younger windblown organic matter. The date on ostrich eggshell fits well with the expected limits of the Bashendi culture.

DOP 92 Subseries

Gd-4844. DOP 92\#1

$8420 \pm 300$

Charcoal from a hearth in red sand, depth 3-4 cm, Grid A, BIIa.

Comment: Small sample, diluted with inactive $\mathrm{CO}_{2}$ for counting.

Gd-6636. DOP 92\#2

$6860 \pm 80$

Charcoal from a hearth, depth 5-6 cm, middle part of Hut I, Grid A, B9c-d.

Gd-6637. DOP 92\#3

$6840 \pm 80$

Charcoal from a hearth in Hut 4, depth $15 \mathrm{~cm}$.

Gd-6645. DOP 92\#4

$6640 \pm 80$

Charcoal from an ashy midden or hearth outside Hut 173 , underlying the surface, depth 3-12 cm, associated with flecks and small chunks, Grid B, TIId.

Gd-6638. DOP 92\#5

$6920 \pm 80$

Charcoal from a hearth within Hut 173, depth $10 \mathrm{~cm}$, Grid B, L12d.

$6990 \pm 70$

Gd-7088. DOP 92\#6

$\delta^{13} C=-5.1 \%$

Ostrich eggshell collected on the surface around stone circles, west side of Grid B. 
Gd-6632. DOP 92\#7

$6650 \pm 80$

Ostrich eggshell from a surface cluster in an activity area within a hut circle, south side of Grid A.

Dakhleh Oasis Lake Subseries

Gd-4618. Dakhleh Oasis DK 8/90

Charcoal, Site 166-c, Square 06/04.

$7030 \pm 240$

Gd-4563. Dakhleh Oasis DK 1/90

Ostrich eggshell, Site 166-c, hearth, Square 05/03.

$8680 \pm 170$

Gd-5712. Dakhleh Oasis DK 7/90

Ostrich eggshell, Site 166-c, Square 06/04.

$\delta^{13} \mathrm{C}=-2.0 \%$

$8180 \pm 70$

$\delta^{13} \mathrm{C}=-2.9 \%$

\section{Qasr-el Sagha Series}

Charcoal from excavations of Neolithic settlements containing remains of the Fayum A culture (Ginter and Kozłowski 1984; Kozłowski and Ginter 1989) in Qasr el-Sagha $\left(30^{\circ} 40^{\prime} \mathrm{N}, 29^{\circ} 20^{\prime} \mathrm{E}\right)$, Western Desert, north of Bisket Lake, southwest of Qasr el-Sagha Temple. Several sites occur within a layer of crossbedded sand from ancient deltaic deposits of Moerris Lake, elevation $100 \mathrm{~m}$ asl (Ginter et al. 1980; Kozłowski 1983; Pazdur 1983). Collected December 1980 and December 1981 and submitted 1981 and 1982 by Bolesław Ginter, Institute of Archeology, Jagellonian University, Kraków.

Gd-903. QS P7/80

From hearth \#1 in white silt layer, Site QS VID/80.

$5410 \pm 110$

Gd-895. QS P10/80

$5070 \pm 110$

From a sandy layer below fossil soil, section 7, Site QS VIIA/80.

Gd-915. QS P10/80A

From a sandy layer below fossil soil, section 6, Site QS VIIA/80.

$5160 \pm 110$

Gd-916. QS P12/80

From a sandy layer above fossil soil, section 6, Site QS VIIA/80.

$5080 \pm 110$

Gd-904. QS P13/80

From a layer of white sand, Trench 1, depth $250-255 \mathrm{~cm}$, Site QS VIII/80.

$5010 \pm 120$

Gd-874. QS P14/80

From hearth \#2, Site QS VIIG/80.

$5120 \pm 110$

Gd-1372. QS P15/80

From a furnace pit, Site QS VIIC/80.

$3890 \pm 45$

Gd-919. QS P16/80

From locus \#2, Site QS VIIA/80.

$5960 \pm 400$

Comment: Small sample, diluted with inactive $\mathrm{CO}_{2}$ for counting.

Gd-980. QS P17/81

From hearth \#5, Site QS X/81.

$6380 \pm 80$

Gd-1499. QS P18/81

From hearth \#1, Site QS IX/81.

$6380 \pm 60$ 
Gd-2021. QS P19/81

$6480 \pm 170$

From hearth \#2, Site QS XI/81.

Gd-1497. QS P20/81

$6320 \pm 60$

From hearth \#1, Site QS X/81.

Gd-979. QS P21/81

$6290 \pm 100$

From hearth \#2, Site QS X/81.

Gd-978. QS P22/81

$4740 \pm 100$

From hearth \#3, Site QS X/81.

Gd-1495. QS P23/81

$5650 \pm 70$

From a hearth in wadi silt, near Site QS VIE/81.

Gd-977. QS P24/81

$5450 \pm 100$

From layer of white sand below soil level, section 8, Site QS VIIA/81.

Gd-1496. QS P25/81

$5000 \pm 60$

From a layer of yellow sand above soil level, section 8, Site QS VIIA/81.

Gd-973. QS P26A/81

$4580 \pm 180$

From a sandy layer with washed hearth, depth $25-30 \mathrm{~cm}$, section 8, Site QS VIIA/81.

Gd-976. QS P26B/81

$4820 \pm 100$

From a sandy layer with dispersed charcoal, depth $30 \mathrm{~cm}$, section 8, Site QS VIIA/81.

Gd-971. QS P27/81

$3190 \pm 130$

From a hearth, Site QS VIIG/81.

Gd-1486. QS P28/81-1/81-A

$3460 \pm 50$

From Feature 1/81, Site QS VIA/81.

Gd-969. QS P28/81-1/81-B

$3430 \pm 60$

From Feature 1/81, duplicate run on the same sample.

Gd-970. QS P28/81-2/81

$3580 \pm 60$

From Feature 2/81, Site QS VIA/81.

Comment (M.F.P.): For a list of previously obtained dates from Qasr el-Sagha, see Pazdur et al. (1982). Kozłowski and Ginter (1989) discussed the stratigraphy of the sites and evaluated the significance of the whole set of dates.

\section{Malkata Armant Series}

Charcoal and wood from excavations of several sites of Predynastic settlement at Malkata Armant (Ginter and Kozłowski 1994) with finds of the Nagadian culture, situated on a sand-and-gravel terrace over the Nile valley, eastern boundary of Western Desert, Egypt. Site MA-2/83 $\left(25^{\circ} 40^{\prime} \mathrm{N}\right.$, $\left.32^{\circ} 35^{\prime} \mathrm{E}\right)$, elevation $110 \mathrm{~m}$ asl; Site MA-6/83 $\left(25^{\circ} 45^{\prime} \mathrm{N}, 32^{\circ} 35^{\prime} \mathrm{E}\right)$, elevation $110 \mathrm{~m}$ asl; Site MA-17/ $83\left(25^{\circ} 40^{\prime} \mathrm{N}, 32^{\circ} 35^{\prime} \mathrm{E}\right)$, elevation $120 \mathrm{~m}$ asl; Site MA- $18 / 83\left(25^{\circ} 45^{\prime} \mathrm{N}, 32^{\circ} 35^{\prime} \mathrm{E}\right)$, elevation $130 \mathrm{~m}$ asl; Site MA-21/83 and Site MA-21A/83 ( $\left.25^{\circ} 45^{\prime} \mathrm{N}, 32^{\circ} 35^{\prime} \mathrm{E}\right)$, elevation $130 \mathrm{~m}$ asl. Collected 19831988 and submitted 1984-1989 by B. Ginter and J. K. Kozłowski, Institute of Archaeology, Jagellonian University, Kraków.

Gd-1756. MA-2/83 \#1/83

$6310 \pm 80$

Charcoal, from a pit with a hearth, depth $20-30 \mathrm{~cm}$. 
Gd-1754. MA-6/83 \#2/83

Charcoal, from hearth \#1, depth $15-30 \mathrm{~cm}$.

$5560 \pm 80$

Gd-3065. MA-17/83 \#3/83

$5140 \pm 60$

Charcoal, dispersed in a cultural layer, depth $10-15 \mathrm{~cm}$.

Gd-3072. MA-18/83 \#4/83

$5090 \pm 50$

Charcoal, from hearth \#1, depth $10-25 \mathrm{~cm}$.

$5030 \pm 60$

Gd-3068. MA-18/83 \#5/83

Charcoal, from hearth \#2, depth $15-25 \mathrm{~cm}$.

$5030 \pm 60$

Gd-5438. MA-6/83S

Freshwater mollusk shell, collected December 1987 by M. Pawlikowski.

$12,270 \pm 120$

$\delta^{13} \mathrm{C}=-4.6 \%$

Site Malkata Armant MA-21/83 Series

Gd-2235. MA-21/83 \#6/83

Charcoal, from a pit with a hearth, depth $25-40 \mathrm{~cm}$.

$5030 \pm 100$

Gd-2985. MA-21/83 Pit \#5, $>25$ cm

Charcoal, from Pit \#5, depth $>25 \mathrm{~cm}$.

$5040 \pm 80$

Gd-3141. MA-21/83 Feature \#5, $<25$ cm

Charcoal, from Feature \#5, depth $<25 \mathrm{~cm}$.

$5020 \pm 50$

Gd-4386. MA-21/83 Pit \#13, 5-15 cm

Charcoal, from Pit \#13, depth 5-15 cm.

$5160 \pm 120$

Gd-1860. MA-21/83 Feature \#26

Charcoal, from Feature \#26.

$4890 \pm 50$

Gd-1979. MA-21/83 Feature \#26, 20-25 cm

Charcoal, below Feature \#26, depth 20-25 cm.

$4920 \pm 90$

Gd-5469. MA-21/83 Feature \#27, 15 cm

$5180 \pm 50$

Charcoal, from a hearth, Feature \#27, depth $15 \mathrm{~cm}$.

$4970 \pm 50$

. MA-21/83 Feature \#33, 5-15 cm

Charcoal, from Feature \#33, depth 5-15 cm.

Gd-3203. MA-21/83 Feature \#33, 5-10 cm

Charcoal, from Feature \#33, depth 5-10 cm.

$4970 \pm 40$

Gd-1862. MA-21/83 Feature \#34a, 32-36 cm

Charcoal, from Feature \#34a, depth $32-36 \mathrm{~cm}$.

$5100 \pm 60$

Gd-1856. MA-21/83 Feature \#34c

Charcoal, from Feature \#34c.

$5190 \pm 50$

Gd-3140. MA-21/83 Feature \#34d, $<17 \mathrm{~cm}$

Charcoal, from Feature \#34d, depth $<17 \mathrm{~cm}$.

$5140 \pm 40$

Gd-2346. MA-21/83 Feature \#34e, $<17 \mathrm{~cm}$

Charcoal, from Feature \#34e, depth $<17 \mathrm{~cm}$.

$4990 \pm 80$

Gd-2986. MA-21/83 Feature \#35, 5-10 cm

Charcoal, from Feature \#35, depth 5-10 cm.

$5200 \pm 90$ 
Gd-2984. MA-21/83 Feature \#35a,10-12 cm $4980 \pm 90$

Charcoal, from Feature \#35a, depth 10-12 cm.

Gd-1925. MA-21/83 Feature \#37, $25-45 \mathrm{~cm}$ $5150 \pm 60$ Charcoal, from Feature \#37, depth $25-45 \mathrm{~cm}$.

Gd-5475. MA-21/83 Feature \#37, >15 cm $4990 \pm 50$ Gd-3439. MA-21/83 Feature \#37, $>15 \mathrm{~cm}$ $4990 \pm 35$

Charcoal, from Feature \#37, depth $>15 \mathrm{~cm}$.

Comment: Independent age determinations on different parts of the same sample using two counting units, mean age $4990 \pm 30 \mathrm{BP}$.

Gd-1980. MA-21/83 Feature \#38, 25-30 cm Charcoal, from Feature \#38, depth $25-30 \mathrm{~cm}$.

Gd-1933. MA-21/83 Feature \#38a, $50 \mathrm{~cm}$ $5070 \pm 80$ Charcoal, from Feature \#38a, depth $20 \mathrm{~cm}$.

Gd-1999. MA-21/83 Feature\#39, 5-15 cm Charcoal, from Feature \#39, depth 5-15 cm.

Gd-2990. MA-21/83 Feature \#40, 15-25 cm $4950 \pm 50$

Gd-5470. MA-21/83 Feature \#40, 15-25 cm $5060 \pm 100$

Gd-3437. MA-21/83 Feature \#40, 15-25 cm Charcoal, from Feature \#40, depth $15-25 \mathrm{~cm}$. $5040 \pm 90$ Comment: Repeated counting of same gas on different counting units; mean age: $5010 \pm 30 \mathrm{BP}$.

Gd-1987. MA-21/83 Feature \#40, $>25 \mathrm{~cm}$ $4830 \pm 70$ Charcoal, from Feature \#40, depth $>25 \mathrm{~cm}$.

Gd-5409. MA-21/83 Feature \#41, 15-25 cm $4930 \pm 60$ Charcoal, from Feature \#41, depth $15-25 \mathrm{~cm}$.

Gd-3268. MA-21/83 Pit $N$ of \#41, $30-40$ cm $4640 \pm 100$ Charcoal, small pit $N$ of Feature \#41, depth $30-40 \mathrm{~cm}$.

Gd-2530. MA-21/83 Feature \#43, 25-35 cm $5010 \pm 100$ Charcoal, from Feature \#43, depth $25-35 \mathrm{~cm}$.

Gd-2529. MA-21/83 Feature \#48 $4710 \pm 100$

Charcoal, from basal part of Feature \#48.

Gd-1981. MA-21/83 Feature \#51, $>30 \mathrm{~cm}$ $4930 \pm 70$ Charcoal, from Feature \#51, depth $>30 \mathrm{~cm}$.

Gd-5408. MA-21/83 Feature \#51, 14-30 cm $4990 \pm 50$ Charcoal, from Feature \#51, depth $14-30 \mathrm{~cm}$.

Gd-5462. MA-21/83 Feature \#53, 10-15 cm $4950 \pm 80$ Charcoal, from Feature \#53, depth $10-15 \mathrm{~cm}$. $5080 \pm 80$

Gd-1993. MA-21/83 Feature \#53a, $>15 \mathrm{~cm}$ Charcoal, from Feature \#53a, depth $>15 \mathrm{~cm}$. 
Gd-3255. MA-21/83 Feature \#53b, $>10 \mathrm{~cm}$ Charcoal, from Feature \#53b, depth $>10 \mathrm{~cm}$.

$4960 \pm 60$

Gd-3275. MA-21/83 Feeature \#53d, $>15 \mathrm{~cm}$ $5080 \pm 60$

Charcoal, from Feature \#53d, depth $>15 \mathrm{~cm}$.

Gd-1857. MA-21/83 Feature \#54, 10-20 cm Charcoal, from Feature \#54, depth $10-20 \mathrm{~cm}$.

Gd-3403. MA-21/83 Feature \#54(?), 20-25 cm

Charcoal, from Feature \#54(?), depth $20-25 \mathrm{~cm}$.

Gd-3144. MA-21/83 Feature \#54a, 25-55 cm Charcoal, from Feature \#54a, depth $25-55 \mathrm{~cm}$.

Gd-3433. MA-21/83 Feature \#54a, $30-40 \mathrm{~cm}$ Charcoal, from Feature \#54a, depth $30-40 \mathrm{~cm}$.

Gd-1998. MA-21/83 Feature \#56, 35-40 cm Charcoal, from Feature \#56, depth $35-40 \mathrm{~cm}$.

Gd-3204. MA-21/83 Feature \#57

Charcoal, from Feature \#57.

Gd-3208. MA-21/83 Feature \#58, 15-35 cm Charcoal, from Feature \#58, depth $15-35 \mathrm{~cm}$.

Gd-3209. MA-21/83 Feature \#58, 40-45 cm Charcoal, from lower part of Feature \#58, depth $40-45 \mathrm{~cm}$.

$4970 \pm 50$

$4940 \pm 50$

$4960 \pm 50$

$4980 \pm 40$

$4690 \pm 80$

$4910 \pm 50$

Gd-3394. MA-21/83 Feature \#59, 10-15 cm

Charcoal, from Feature \#59, depth 10-15 cm.

$4820 \pm 60$

Gd-3434. MA-21/83 Feature \#60, 0-5 cm

Charcoal, from Feature \#60, depth $0-5 \mathrm{~cm}$.

Gd-3404. MA-21/83 Feature \#75, 5-10 cm

Charcoal, from Feature \#75, depth $5-10 \mathrm{~cm}$.

Gd-3435. MA-21/83 Feature \#76, 5-10 cm

Charcoal, from Feature \#76, depth 5-10 cm.

$4960 \pm 50$

Gd-3385. MA-21/83 Feature \#77, 10-15 cm

Charcoal, from Feature \#77, depth $10-15 \mathrm{~cm}$.

$4980 \pm 50$

Gd-2528. MA-21/83 Layer Delta 25-27

$5010 \pm 25$

$5020 \pm 40$

$5050 \pm 25$

Charcoal, from cultural layer delta, depth $25-27 \mathrm{~cm}$.

Gd-3142. MA-21/83 M22, 10-15 cm

Charcoal, from cultural layer at depth $10-15 \mathrm{~cm}$, loc. M22.

$5010 \pm 40$

Gd-3143. MA-21/83 M22, 2-5 cm

Charcoal, from cultural layer at depth $2-5 \mathrm{~cm}$, loc. M22.

$4990 \pm 40$

Gd-2347. MA-21/83 M22, 5-10 cm

Charcoal, from cultural layer at depth 5-10 cm, loc. M22.

$5000 \pm 60$ 
Gd-5459. MA-21/83 A21, 5-20 cm

$4950 \pm 50$

Charcoal, from cultural layer, depth $5-20 \mathrm{~cm}$, loc. A21.

Gd-1859. MA-21/83 B21, 15-20 cm

$5060 \pm 50$

Charcoal, from cultural layer at depth $15-20 \mathrm{~cm}$, loc. B21.

Gd-3139. MA-21/83 B21, 2-5 cm

$4960 \pm 40$

Charcoal, from cultural layer at depth $2-5 \mathrm{~cm}$, loc. B21.

Gd-1858. MA-21/83 B22, 5-15 cm

$4950 \pm 70$

Charcoal, from cultural layer at depth 5-15 cm, loc. B22.

$4920 \pm 60$

Gd-1861. MA-21/83 Cult layer, 22-27 cm

$4920 \pm 60$

Charcoal, from cultural layer, depth $22-27 \mathrm{~cm}$.

Site Malkata Armant MA-21/83 Palisade Series

Highly decomposed wooden piles from the remnants of a palisade.

Gd-2981. MA-21/83 P-303

$5090 \pm 90$

Pile \#303.

Gd-5460. MA-21/83 P-313

$5180 \pm 60$

Pile \#313.

Gd-2977. MA-21/83 P-315

$5140 \pm 90$

Pile \#315.

Gd-2978. MA-21/83 P-316

$5060 \pm 90$

Pile \#316.

Gd-2979. MA-21/83 P-317

$5190 \pm 90$

Pile \#317.

Gd-5461. MA-21/83 P-321

$\mathbf{5 5 0 0} \pm \mathbf{5 0}$

Pile \#321.

Gd-2980. MA-21/83 P-323

$5320 \pm 110$

Pile \#323.

Gd-4378. MA-21/83 P-325

$5220 \pm 90$

Pile \#325.

Site Malkata Armant MA-21A/83 Series

Gd-3395. MA-21A/83 Feature \#201

$4820 \pm 30$

Charcoal, from Feature \#201, PIV-1.

Gd-3400. MA-21A/83 Feature \#215a

$4830 \pm 40$

Charcoal, from Feature \#215a, PIV-2.

Gd-3398. MA-21A/83 Feature \#217

$4790 \pm 35$

Charcoal, from Feature \#217, PIV-3.

Gd-3402. MA-21A/83 Feature \#218a

$4930 \pm 30$

Charcoal, from Feature \#218a, PIV-4. 
Gd-5499. MA-21A/83 Feature \#223a, 20-25 cm $4970 \pm 60$

Charcoal, from Feature \#223a, depth 20-25 cm.

Gd-6015. MA-21A/83 Feature \#232x, 5-10 cm $5070 \pm 110$

Charcoal, from Feature \#232x, depth 5-10 cm.

Gd-5500. MA-21A/83 Feature \#238, 15-20 cm $4970 \pm 60$

Charcoal, from Feature \#238, depth $15-20 \mathrm{~cm}$.

Gd-5501. MA-21A/83 Feature \#238a, $25-30 \mathrm{~cm}$

$4960 \pm 50$

Charcoal, from Feature \#238a, depth $25-30 \mathrm{~cm}$.

Gd-3450. MA-21A/83 Feature \#238b, 35-40 cm $5075 \pm 25$ Charcoal, from Feature \#238b, depth $35-40 \mathrm{~cm}$.

Gd-5502. MA-21A/83 Feature \#252, $15-30 \mathrm{~cm}$ Charcoal, from Feature \#252, depth $15-30 \mathrm{~cm}$.

Gd-5503. MA-21A/83 Feature \#253, 25-40 cm Charcoal, from Feature \#253, depth $25-40 \mathrm{~cm}$.

$\delta^{13} C=-26.0 \%$ $4790 \pm 60$

Gd-3432. MA-21A/83 Feature \#256a

Charcoal, from Feature \#256a.

$4890 \pm 60$

Gd-3427. MA-21A/83 Feature \#257

Charcoal, from Feature \#257.

$5060 \pm 35$

$\delta^{13} C=-27.1 \%$

$5090 \pm 60$

Gd-2925. MA-21A/83 Alfa03, 5-10 cm

$\delta^{13} C=-28.1 \%$

$4910 \pm 80$

Charcoal, from cultural layer, depth $5-10 \mathrm{~cm}$, loc. z/alfa 03 .

Gd-5416. MA-21A/83 Alfa03, 50-70 cm

$5160 \pm 50$

Charcoal, from cultural layer, depth $50-70 \mathrm{~cm}$, loc. z/alfa 03 .

$4990 \pm 50$

Charcoal, from cultural layer, depth $20-25 \mathrm{~cm}$, loc. beta 02 .

Gd-3431. MA-21A/83 Beta02, 30-35 cm

Charcoal, from cultural layer, depth $30-35 \mathrm{~cm}$, loc. beta 02 .

$4990 \pm 35$

$\delta^{13} \mathrm{C}=-27.1 \%$ 。

Gd-3428. MA-21A/83 Beta02, $>60 \mathrm{~cm}$

Charcoal, from cultural layer, depth $>60 \mathrm{~cm}$, loc. beta 02 .

$5050 \pm 70$

$\delta^{13} C=-26.3 \%$

Comment (M.F.P.): Correlation of ${ }^{14} \mathrm{C}$ dates with associated finds and site stratigraphy enables the assignment of precise time limits to phases of development of Predynastic settlements at Malkata Armant. The floruit of the oldest phase (A) at Site MA-21/83, defined by the interquartile range of the composite probability distribution of the appropriate set of five ${ }^{14} \mathrm{C}$ dates, is confined between 4040 and $3910 \mathrm{cal} \mathrm{BC}$, with a midpoint at $3980 \mathrm{cal}$ BC. The duration of the middle phase (B) at Site MA$21 / 83$, based on the set of 21 dates, was $\sim 3910-3760 \mathrm{cal} \mathrm{BC}$, with a midpoint at $3840 \mathrm{cal} \mathrm{BC}$. The duration of the youngest phase (C) at Site MA-21/83, based on the set of 21 dates, was $\sim 3840-3720 \mathrm{cal}$ $\mathrm{BC}$, with a midpoint at $3760 \mathrm{cal} \mathrm{BC}$. Corresponding analysis of results obtained for Site MA-21A/83 yielded the following estimates: phase A (1 date): floruit $4000-3940 \mathrm{cal} \mathrm{BC}$, midpoint $3980 \mathrm{cal} \mathrm{BC}$; phase B ( 5 dates): floruit $3900-3770$ cal BC, midpoint 3840 cal BC; phase $C$ ( 7 dates): floruit 3760 $3650 \mathrm{cal} \mathrm{BC}$, midpoint $3710 \mathrm{BC}$. Calculation of the composite probability distribution of the set of 7 dates obtained on wood samples from the palisade remains found at Site MA-21/83 yields a midpoint of $3990 \mathrm{cal} \mathrm{BC}$, with uncertainty determined by the interquartile range $4100-3880 \mathrm{cal} \mathrm{BC} .{ }^{14} \mathrm{C}$ dates 
were calibrated according to the procedure described by Pazdur and Michczyńska $(1989,1993)$; Pazdur $e$ al. (1994) discuss in detail the ${ }^{14} \mathrm{C}$ dates obtained for the settlements at Malkata Armant.

\section{Uan Muhuggiag Series}

Seeds, fruits and other macroscopic plant fragments from Uan Muhuggiag Rockshelter, located in the Central Acacus, Tadrart Acacus area, northern side of Wadi Teshuinat, North Sahara, Libya. Collected 1982 by B. E. Barich during the Libyan-Italian Joint Mission for Saharan Research; submitted April 1988 by K. Wasylikowa, Institute of Botany, Polish Academy of Sciences, Kraków. General characteristics of the site and its relevance to the late prehistory of the Libyan Sahara are described by Barich $(1974,1984,1989) ;{ }^{14} \mathrm{C}$ dates from the site previously obtained are discussed by Barich et al. (1984); present results are discussed by Pazdur (1993).

Gd-4290. UAM B1/Citr

Seeds of Citrullus colocynthis from Sector B, Level 1.

$2220 \pm 220$

Gd-4288. UAM B1/Copr

Coprolites from Sector B, Level 1.

$\delta^{13} \mathrm{C}=-25.0 \%$

Gd-2854. UAM B1/Bal

Fruits of Balanites aegyptiaca from Sector B, Level 1.

$2770 \pm 80$

$\delta^{13} C=-21.0 \%$

Gd-5337. UAM B2b

Fruits of Balanites aegyptiaca from Sector B, Level $2 \mathrm{~b}$.

$3810 \pm 80$

$\delta^{13} C=-23.4 \%$

Gd-2853. UAM A2a

Coprolites from Sector A, Level 2a.

$5420 \pm 50$

$\delta^{13} \mathrm{C}=-24.4 \%$ o

$6030 \pm 80$

$\delta^{13} C=-21.7 \%$

Gd-2962. UAM A1a

$3720 \pm 90$

Kernels of Balanites sp. and other plant fragments from Sector A, Level 1a.

$\delta^{13} \mathrm{C}=-25.5 \%$

Gd-4363. UAM A1a-bis

Repeated run on the same sample.

$3800 \pm 140$

$\delta^{13} C=-25.5 \%$

Gd-4358. UAM A2c

Kernels of Balanites aegyptiaca from Sector A, Level 1a.

$5780 \pm 80$

$\delta^{13} C=-24.1 \%$

Gd-4362. UAM A2

Kernels of Balanites aegyptiaca from Sector A, Level 2.

$5290 \pm 110$

Gd-2959. UAM B2

Kernels of Balanites aegyptiaca from Sector B, Level 2.

$\delta^{13} \mathrm{C}=-24.0 \%$

Gd-2960. UAM B2a

Kernels of Balanites aegyptiaca from Sector B, Level 2a.

$5340 \pm 120$

$\delta^{13} C=-24.4 \% 0$

$5420 \pm 100$

$\delta^{13} C=-25.0 \%$

Gd-4361. UAM B2a-bis

$5480 \pm 120$

Repeated run on the same sample.

$\delta^{13} C=-25.0 \%$

$5210 \pm 90$

Gd-2855. TH2/I

Plant fragments from layer I, Site 2, in Ti-n-Torha (Barich 1974, 1984).

Gd-926. BK-E-79-4

$6330 \pm 100$

Charcoal from a hearth below stony plates in a layer of silts in Bir Kiseiba, ca. $150 \mathrm{~km}$ west of Assuan, Western Desert, Egypt $\left(23^{\circ} \mathrm{N}, 30^{\circ} \mathrm{E}\right)$. The site is located at elevation $200 \mathrm{~m}$ asl on the border 
of a dry shallow water basin (playa). Collected 1980 and submitted 1981 by Michał Kobusiewicz, Institute of History of Material Culture, Polish Academy of Sciences, Poznan.

\section{Kadero series}

The Neolithic site at Kadero $\left(15^{\circ} 45^{\prime} \mathrm{N}, 32^{\circ} 36^{\prime} \mathrm{E}\right)$, Khartoum Province, Sudan, is located on a low eroded mound of sand which rises ca. $1.8 \mathrm{~m}$ above the flat bottom of the main Nile valley floor. The site is $18 \mathrm{~km}$ north of the confluence of the White and Blue Niles, $6.5 \mathrm{~km}$ east of the channel of the main Nile. Excavations of the site were started in 1972 and resulted in discovery and detailed examination of two settlements and burial grounds (Krzyżaniak 1984). The studies undertaken involve subsistence economy based on food remains excavated from the southern settlement (Krzyżaniak 1978), lithic industry (Nowakowski 1984), pottery (Chłodnicki 1984), archeozoology (Gautier, 1984 ) and archeobotany (Klichowska 1978, 1984). Separate studies were devoted to cemeteries discovered close to the Kadero settlement (Dzierżykray-Rogalski 1984; Prominska 1984). Shell and charred bones were collected from the northern settlement and a burial ground in 1987 and 1989 and submitted by Lech Krzyżaniak, Archaeological Museum, Poznań.

Gd-5653. Kadero 87/1

Single shell of Nile oyster Etheria elliptica, Unit C-65/66, depth $10 \mathrm{~cm}$.

Gd-5651. Kadero 87/2A

Shells of Etheria elliptica, 3 fragments, Unit C-65/66, depth 10-20 cm.

Gd-6164. Kadero 87/2B

Shells of Nile bivalve Aspatharia rubens, 2 fragments, same locality.

Gd-5649. Kadero 87/3

Shells of Aspatharia rubens, Unit C-65/66/67, depth $20-40 \mathrm{~cm}$.

Gd-6165. Kadero 87/4A

Shells of swamp snail Pila ovata, 5 fragments, Unit C-67/68, depth $0-40 \mathrm{~cm}$.

Gd-5652. Kadero 87/4B

Shells of Aspatharia rubens, 10 fragments, same locality.

Gd-5648. Kadero 87/5A

Shells of Pila ovata, Unit C-67/68, depth $0-40 \mathrm{~cm}$.

Gd-6161. Kadero 87/5B

Shells of Aspatharia rubens, same locality.

Gd-5650. Kadero 89/1

Shells of land snail Limmicolaria flammata, Unit C-75/76, depth 0-30 cm.

Gd-5647. Kadero 89/2

Shell of Etheria elliptica, single fragment, Unit C-75/76, depth 10-20 cm.

Gd-6198. Kadero 89/3

Burned animal bones, Unit C-75/76, depth $10-20 \mathrm{~cm}$.

Gd-6167. Kadero 89/4

Shells of Aspatharia rubens, 6 fragments, base of Grave 114, depth $70 \mathrm{~cm}$.

$$
\begin{array}{r}
\mathbf{5 4 5 0} \pm \mathbf{7 0} \\
\delta^{13} C=-4.9 \% 0 \\
\mathbf{5 3 7 0} \pm \mathbf{6 0} \\
\delta^{13} C=-5.1 \% \\
\mathbf{5 5 1 0} \pm \mathbf{1 2 0} \\
\delta^{13} C=-3.3 \% \circ \\
\mathbf{5 4 3 0} \pm \mathbf{6 0 0} \\
\delta^{13} C=-4.0 \% 0 \\
\mathbf{5 7 7 0} \pm \mathbf{1 0 0} \\
\delta^{13} C=-5.6 \% 0 \\
\mathbf{5 4 2 0} \pm \mathbf{7 0} \\
\delta^{13} C=-3.2 \% 0 \\
\mathbf{5 7 2 0} \pm \mathbf{5 0} \\
\delta^{13} C=-4.8 \% 0 \\
\mathbf{5 6 9 0} \pm \mathbf{8 0} \\
\delta^{13} C=-1.7 \% \\
\mathbf{5 4 8 0} \pm \mathbf{6 0} \\
\delta^{13} C=-7.5 \% \\
\mathbf{5 9 6 0} \pm \mathbf{7 0} \\
\delta^{13} C=-11.4 \% \\
\mathbf{5 3 9 0} \pm \mathbf{9 0} \\
\delta^{13} C=-21.1 \% \\
\mathbf{5 5 1 0} \pm \mathbf{1 0 0} \\
\delta^{13} C=-4.4 \% \circ
\end{array}
$$

Comment (L.K.): Grave pit with well-defined boundaries, containing human remains with furniture. 
Gd-6162. Kadero 89/5

$5260 \pm 120$

Shells of Nile bivalve Aspatharia rubens, from Grave 101, depth $50 \mathrm{~cm}$.

$\delta^{13} \mathrm{C}=-4.4 \%$

Comment (L.K.): Grave pit boundaries not visible; grave contains human remains with furniture.

\section{Minshat Abu Omar Series}

Charcoal and shell from excavations undertaken by Munich East-Delta Expedition (MOE) under the direction of Dietrich Wildung on a Late Predynastic-Early Dynastic cemetery situated in the Eastern Nile Delta, north of modern village Minshat Abu Omar $\left(30^{\circ} 55^{\prime} \mathrm{N}, 32^{\circ} 02^{\prime} \mathrm{E}\right), \mathrm{ca} .30 \mathrm{~km}$ northeast of Faqus. According to Wildung (1984), the cemetery was used between Nagada II and the First Dynasty and then again in the Roman period. Kroeper (1984) summarized the results of the first stage of MOE activities in Minshat Abu Omar; Krzyżaniak (1989) presented comparative analysis of pottery and other finds from several sites in the study area. Collected and submitted 1990 by Lech Krzyżaniak, Archaeological Museum, Poznań.

Gd-6233. MAO 1990/1

$3930 \pm 70$

Charcoal and charred plant remains from wooden construction of the chamber of Grave 1590.

Gd-4566. MAO 1990/2

$4120 \pm 100$

Charred plant remains from the contents of funerary ceramic vessels 7-9 found in Grave 1930.

Gd-5713. MAO 1990/3

$5240 \pm 60$

$\delta^{13} C=-6.1 \%$

Shell of a river bivalve from depth $2.5 \mathrm{~m}$ below the surface of sandy hill (gezira), near Grave 1930, from pure sand.

Gd-6232. MAO 1990/4

$9000 \pm 110$

Shell of land snail Helicidae, Square 13/21-20, pure sandy layer, depth 1-2 m.

$\delta^{13} C=-5.6 \%$

IRON AGE

Dongola Series

Charcoal from the excavation of a graveyard in Old Dongola, Northern Province, Egypt $\left(18^{\circ} 13^{\prime} \mathrm{N}\right.$, $\left.30^{\circ} 45^{\prime} \mathrm{E}\right)$. Collected February 1989 and submitted 1989 by Bogusław Zurawski, Department of Mediterranean Archaeology, Polish Academy of Sciences, Warsaw.

Gd-5666. Dongola I/89

$1270 \pm 30$

From a "lamp box" made of two bricks, above a grave pit, TEQ I-2, depth $15 \mathrm{~cm}$.

Gd-3486. Dongola II/89

From a kiln, depth $75 \mathrm{~cm}$.

$1120 \pm 50$

$\delta^{13} C=-24.5 \%$

Gd-6180. Dongola IV/89

$1020 \pm 45$

From a "lamp box" at the west wall of a grave, TWH IV, depth $35 \mathrm{~cm}$.

Gd-6179. Dongola XII/89

$1090 \pm 60$

From a burial chamber, TSJ-2, depth $175 \mathrm{~cm}$.

Gd-5405. Dongola II/88

$1360 \pm 40$

Wood, fragment of a board from the altar of a Crusader Church found at depth $5 \mathrm{~m}$ in Dongola, Northern Province, Egypt $\left(19^{\circ} \mathrm{N}, 30^{\circ} \mathrm{E}\right)$. Collected 1987 and submitted 1988 by Władysław Godlewski, National Museum, Warsaw. 
Gd-5450. Dongola II/88bis

Repeated run on the same sample.

$1400 \pm 45$

Comment (M.F.P.) ${ }^{14} \mathrm{C}$ dates of two parts of the same sample predate the first Crusade by several centuries; the old wood effect seems a reasonable explanation.

Gd-3417. Tell Atrib IA

$1770 \pm 30$

Charcoal, scattered within a ca. 1 -m-thick layer consisting of rubble (ash, mortar and marble, with numerous fragments of pottery, glass and bronze), depth 2-3 m, Sector T of mound Kon Sidi Youssef, Tell Atrib, in Benha (ancient Athribis), $\left(30^{\circ} 25^{\prime} \mathrm{N}, 31^{\circ} 10^{\prime} \mathrm{E}\right)$, Lower Egypt, floor of Nile valley, elevation $2 \mathrm{~m}$ asl. Collected November 1981 and submitted 1988 by T. Gorecki, National Museum, Warsaw.

\section{Asantemanso Series}

Charcoal from excavations in Asantemanso, district Asante $\left(6^{\circ} 30^{\prime} \mathrm{N}, 1^{\circ} 30^{\prime} \mathrm{W}\right)$, Adansemanso, district Adanse $\left(6^{\circ} 17^{\prime} \mathrm{N}, 1^{\circ} 35^{\prime} \mathrm{W}\right)$, in Anyinam, district Amansie East $\left(6^{\circ} 30^{\prime} \mathrm{N}, 1^{\circ} 32^{\prime} \mathrm{W}\right)$, and in Esiease, district Amansie East $\left(6^{\circ} 28^{\prime} \mathrm{N}, 1^{\circ} 31^{\prime} \mathrm{W}\right)$, Ghana, Central Africa. Collected 1989, 1990 and 1991 and submitted 1990 and 1991 by P. L. Shinnie, Department of Archaeology, University of Calgary, Alberta, Canada.

Gd-6330. AS06-C55

Trench 6, cultural layer, depth $20-30 \mathrm{~cm}$.

$410 \pm 60$

Gd-5807. AS07-C56

Trench 7, cultural layer, depth $80-90 \mathrm{~cm}$.

$710 \pm 50$

Gd-6326. AS08-C65

Trench 8, cultural layer, depth $80-90 \mathrm{~cm}$, associated with iron slag.

$410 \pm 80$

Gd-5798. AS08-C67

Trench 8, cultural layer, depth 50-60 cm, mixed with iron slag and ceramics.

$810 \pm 40$

Gd-5806. AS09-C69

Carbonized palm nuts from Trench 9, cultural layer, depth $30-40 \mathrm{~cm}$.

$440 \pm 40$

Gd-5801. AS10-C71

Trench 10, cultural layer, depth $40-60 \mathrm{~cm}$.

$2440 \pm 60$

Gd-5804. AS10-C70

Trench 10, cultural layer, depth $40-60 \mathrm{~cm}$.

$2480 \pm 60$

Gd-6327. AS11-C74

Trench 11, cultural layer, depth $100-110 \mathrm{~cm}$.

$420 \pm 70$

Gd-4644. AS12-C90

Trench 12, pit feature, depth $155-177 \mathrm{~cm}$.

Modern

Gd-5805. AS12-C91

Trench 12, cultural layer, depth $40-50 \mathrm{~cm}$.

$\mathbf{3 0 0} \pm \mathbf{5 0}$

Gd-6329. AS13-C92

Trench 13, cultural layer, depth $60-70 \mathrm{~cm}$, associated with pipe fragments.

Modern

Gd-6328. AS13-C93

Trench 13, cultural layer, depth $60-70 \mathrm{~cm}$, associated with pipe fragments.

$240 \pm 70$ 
Gd-5802. AS15-C101

$480 \pm 40$

Trench 15, cultural layer, depth $30-40 \mathrm{~cm}$.

Gd-5803. AS10-C118

$\mathbf{5 9 0} \pm \mathbf{5 0}$

Trench 10, cultural layer, depth $60-80 \mathrm{~cm}$.

Gd-5800. AS27-C140

$470 \pm 50$

Trench 27, cultural layer, depth $20-40 \mathrm{~cm}$.

Gd-6331. AS56-C173

$640 \pm 80$

Trench 56, depth 50-60 cm.

\section{Adansemanso Series}

Gd-6540. AD-C201

$310 \pm 60$

Base of cultural unit, depth $40-50 \mathrm{~cm}$, associated with two glass beads, Trench A5.

Gd-5996. AD-C210

$680 \pm 50$

Cultural layer below floor level, depth $130-140 \mathrm{~cm}$, Trench B14.

Gd-6545. AD-C214

$740 \pm 80$

Inside furnace structure in context with iron slag, Feature 2, Mound C, depth 130-140 cm.

Gd-6541. AD-C227

$1050 \pm 100$

Mound C, cultural layer at depth $70-80 \mathrm{~cm}$.

Gd-6537. AD-C234

$1110 \pm 100$

Depth $50 \mathrm{~cm}$, Trench St3.

\section{Anyinam Series}

Gd-6546. AN-C236

$170 \pm 80$

Depth $50 \mathrm{~cm}$.

Gd-5998. AN-C239

$\mathbf{5 5 0} \pm \mathbf{5 0}$

Bottom of deep pit feature within house mound A, depth $310 \mathrm{~cm}$, associated with European imports, Trench AyA.

\section{Esiease Series}

Gd-5997. ES-C262

$230 \pm 50$

From cultural layer at depth $70-80 \mathrm{~cm}$, associated with European imports, Trench EsC.

Gd-6543. ES-C265

$190 \pm 80$

From cultural layer at depth $80-90 \mathrm{~cm}$, associated with European imports, Trench EsD.

Gd-6542. ES-C271

$450 \pm 100$

From cultural layer at depth $80-90 \mathrm{~cm}$, associated with local pipes, Trench EsH.

\section{Niani Series}

Organic detritus, partly charred, from wooden-clayey building destroyed by fire, probably the palace of the King of Mali, Site \#1, Palace $\left(11^{\circ} 22^{\prime} \mathrm{N}, 8^{\circ} 23^{\prime} \mathrm{E}\right)$, in the royal quarter, Niani, near Sankarani, West Africa (Filipowiak 1977; 1981). Collected March 1973 and submitted 1984 by W. Filipowiak, National Museum, Szczecin. 
Gd-2194. Niani \#6/73

From a fire layer in the northeast corner of the palace, below a layer of clay formed by decomposition of air-dried bricks (banco) of local origin, depth $15 \mathrm{~cm}$, associated with baked and dried clay, stones and pottery.

Gd-2195. Niani \#9/73

From a fire layer in banco clay, at the destroyed west wall of the palace, depth $32 \mathrm{~cm}$.

Comment (W.F. and M.F.P.): Date of sample \#6/73 is rejuvenated, probably by mechanical contamination with recent material. Date of sample \#9/73 agrees well with other ${ }^{14} \mathrm{C}$ dates obtained for Site 1 in the royal quarter: KI-292: $380 \pm 50 \mathrm{BP}$; Gif-915: $300 \pm 90 \mathrm{BP}$.

\section{Bir Safsaf Series}

Partly decomposed wood from a large mound southwest of Bir Safsaf, Western Desert, Egypt. No association with definite cultural layer excavated at the site. Samples collected 1992 and submitted by R. Schild to check the rate of degradation of subfossil wood in specific conditions of desert sand cover.

Gd-7202. Bir Safsaf 7/92

$890 \pm 40$

Gd-7208. Bir Safsaf 8/92

$1350 \pm 50$

\section{REFERENCES}

Barich, B. E. 1974 La serie stratigrafica dell Uadi Ti-nTorha. Per una interpretazione delle facies a ceramica saharo-sudanesi. Origini 8: 7-184.

1984 The Epipalaeolithic-ceramic groups of Libyan Sahara: Notes for an economic model of the cultural development in the West-Central Sahara. In Krzyża niak, L. and Kobusiewicz, M., eds., Origin and Early Development of Food-Producing Cultures in NorthEastern Africa. Poznań, Polish Academy of Sciences and Poznań Archaeological Museum: 399-410.

1989 Uan Muhuggiag rock shelter (Tadrart Acacus) and the late prehistory of the Libyan Sahara. In Krzyżaniak, L. and Kobusiewicz, M., eds., Late Prehistory of the Nile Basin and the Sahara. Poznań, Poznań Archaeological Museum: 499-505.

Barich, B. E., Belluomini, G., Bonadonna, F., Alessio, M. and Manfra, L. 1984 Ecological and cultural relevance of the recent new radiocarbon dates from Libyan Sahara. In Krzyżaniak, L. and Kobusiewicz, M., eds., Origin and Early Development of Food-Producing Cultures in North-Eastern Africa. Poznań, Polish Academy of Sciences and Poznań Archaeological Museum: 411-417.

Bluszcz, A. and Pazdur, M. F. 1986 TL and ${ }^{14} \mathrm{C}$ dating of the Upper Palaeolithic site at Wadi Kubbaniya, Egypt. Acta Interdisciplinaria Archaeologica 4: 97-105. 1987 Thermoluminescence dating of the Middle Paleolithic at Wadi Kubbaniya. In Wendorf, F., Schild, R. and Close, A., eds., The Prehistory of Wadi Kubbaniya, Vol. 2. Stratigraphy, Paleoeconomy, and Environment. Dallas, Texas, Southern Methodist University Press: 270-273.
Brookes, I. 1983 Dakhleh Oasis-A geoarchaeological reconnaissance. Journal of the Society for the Study of Egyptian Antiquities 13: 167-177.

1989 Early Holocene basinal sediments of the Dakhleh Oasis Region, South Central Egypt. Quaternary Research 32: 139-152.

Chłodnicki, M. 1984 Pottery from the Neolithic settlement at Kadero (Central Sudan). In Krzyżaniak, L. and Kobusiewicz, M., eds., Origin and Early Development of Food-Producing Cultures in North-Eastern Africa. Poznań, Polish Academy of Sciences and Poznań Archaeological Museum: 337-342.

Donahue, D. J., Jull, A. J. T., Linick, T. W. and Zabel, T. 1987 AMS radiocarbon measurements on material from Wadi Kubbaniya. In Wendorf, F., Schild, R. and Close, A., eds., The Prehistory of Wadi Kubbaniya, Vol. 2. Stratigraphy, Paleoeconomy, and Environment. Dallas, Texas, Southern Methodist University Press: 280-283.

Dzierżykray-Rogalski, T. 1984 Remarks on the position of human remains in the Neolithic graves at Kadero (Central Sudan). In Krzyżaniak, L. and Kobusiewicz, M., eds., Origin and Early Development of Food-Producing Cultures in North-Eastern Africa. Poznań, Polish Academy of Sciences and Poznań Archaeological Museum: 333-335.

Edwards, I. and Hope, C. A. 1989 A note on the Neolithic ceramics from the Dakhleh Oasis (Egypt). In Krzyża niak, L. and Kobusiewicz, M., eds., Late prehistory of the Nile Basin and the Sahara. Poznań, Poznań Archaeological Museum: 233-242. 
Filipowiak, W. 1977 Results of archaeological research of Niani. Nyame Akuma. Newsletter of African Archaeology 11: 32-33.

1981 Niani: Capital of the Mali Kingdom in the 6th17 th Centuries. Wroclaw-Warsaw-Gdansk-Kraków, Ossolineum, 302 p.

Gautier, A. 1984 The fauna of the Neolithic site of Kadero (Central Sudan). In Krzyżaniak, L. and Kobusiewicz, M., eds., Origin and Early Development of Food-Producing Cultures in North-Eastern Africa. Poznań, Polish Academy of Sciences and Poznań Archaeological Museum: 317-319.

Ginter, B., Heflik, W., Kozłowski, J. K. and Śliwa, J. 1980 Excavations in the region of Qasr el-Sagha, 1979. Mitteilungen des Deutschen Archaeologischen Instituts, Abteilung Kairo 36: 105-169.

Ginter, B. and Kozłowski, J. K. 1984 Tarifian and the problem of the origin of Nagadian. In Krzyżaniak, L. and Kobusiewicz, M., eds., Origin and Early Development of Food-Producing Cultures in North-Eastern Africa. Poznań, Polish Academy of Sciences and Poznań Archaeological Museum: 247-260.

eds. 1994 Predynastic Settlements near Armant Sites 21 and 21A. Heidelberg, Heidelberger Orientaler Verlag.

Haas, H. 1987 The radiocarbon dates from Wadi Kubbaniya. In Wendorf, F., Schild, R. and Close, A., eds., The Prehistory of Wadi Kubbaniya, Vol. 2. Stratigraphy, Paleoeconomy, and Environment. Dallas, Texas, Southern Methodist University Press: 274-279.

Hedges, R. E. M., Housley, R. A., Bronk Ramsey, C. R. and van Klinken, G. J. 1993 Radiocarbon dates from the Oxford AMS system: Archaeometry datelist 16. Archaeometry 35(1): 147-167.

Hietala, H. J. 1987 Contemporaneity and occupational duration of the Kubbaniyan sites: An analysis and interpretation of the radiocarbon dates. In Wendorf, F., Schild, R. and Close, A., eds., The Prehistory of Wadi Kubbaniya, Vol. 2. Stratigraphy, Paleoeconomy, and Environment. Dallas, Texas, Southern Methodist University Press: 284-291.

Klichowska, M. 1978 Preliminary results of palaeoethnobotanical studies on plant impressions on potsherds from the Neolithic settlement at Kadero. Nyame Akuma. Newsletter of African Archaeology 12: 42-43.

1984 Plants of the Neolithic Kadero (Central Sudan): A palaeoethnobotanical study of the plant impressions on pottery. In Krzyżaniak, L. and Kobusiewicz, M., eds., Origin and Early Development of Food-Producing Cultures in North-Eastern Africa. Poznań, Polish Academy of Sciences and Poznań Archaeological Museum: 321-326.

Kozłowski, J. K., ed. 1983 Qasr el-Sagha 1980. Contributions to the Holocene geology, the Predynastic and Dynastic settlements in the Northern Fayum Desert. Zeszyty Naukowe Uniwersytetu Jagiellonskiego, Prace Archeologiczne 35: 7-123.
Kozłowski, J. K. and Ginter, B. 1989 The Fayum Neolithic in the light of new discoveries. In Krzyżaniak, L. and Kobusiewicz, M., eds., Late Prehistory of the Nile Basin and the Sahara. Poznań, Poznań Archaeological Museum: 157-179.

Kroeper, K. 1984 Minshat Abu Omar (Munich East Delta Expedition). Bulletin de Liason du Groupe International d'étude de la Céramique Égyptienne 9: 6-10.

Krzyżaniak, L. 1978 New light on early food-production in the Central Sudan. Journal of African History 19: 159-172.

1984 The Neolithic habitation at Kadero (Central Sudan). In Krzyżaniak, L. and Kobusiewicz, M., eds., Origin and Early Development of Food-Producing Cultures in North-Eastern Africa. Poznań, Polish Academy of Sciences and Poznań Archaeological Museum: 309-315.

Krzyżaniak, L. 1989 Recent archaeological evidence on the earliest settlement in the eastern Nile Delta. In Krzyżaniak, L. and Kobusiewicz, M., eds., Late Prehistory of the Nile Basin and the Sahara. Poznań, Poznań Archaeological Museum: 267-285.

Krzyżaniak, L. and Kobusiewicz, M., eds., 1984 Origin and Early Development of Food-Producing Cultures in North-Eastern Africa. Poznań, Polish Academy of Sciences and Poznań Archaeological Museum: 504 p. 1989 Late Prehistory of the Nile Basin and the Sahara. Poznań, Poznań Archaeological Museum: $547 \mathrm{p}$. 1993 Environmental Change and Human Culture in the Nile Basin and Northern Africa Until the 2nd Millenium BC. Poznań, Poznań Archeological Museum.

McDonald, M. M. A. 1990 New evidence from the early to mid-Holocene in Dakhleh Oasis, South-Central Egypt, bearing on the evolution of cattle pastoralism. Nyame Akuma. Newsletter of African Archaeology 33: 3-9.

Mills, A. J. 1984 Research in the Dakhleh Oasis. In Krzyżaniak, L. and Kobusiewicz, M., eds., Origin and Early Development of Food-Producing Cultures in North-Eastern Africa. Poznań, Polish Academy of Sciences and Poznań Archaeological Museum: 205210.

Nowakowski, J. 1984 The typology of lithic implements from the Neolithic settlement at Kadero (Central Sudan). In Krzyżaniak, L. and Kobusiewicz, M., eds., Origin and Early Development of Food-Producing Cultures in North-Eastern Africa. Poznań, Polish Academy of Sciences and Poznań Archaeological Museum: 343-351.

Pazdur, A., Awsiuk, R., Bluszcz, A., Pazdur, M. F., Walanus, A. and Zastawny, A. 1982 Gliwice radiocarbon dates VII. Radiocarbon 24(2): 171-181.

Pazdur, M. F. 1983 Radiocarbon dating of organic samples. In Kozłowski, J. K., ed., Qasr el-Sagha 1980. Contributions to the Holocene geology, the Predynastic and Dynastic settlements in the Northern Fayum 
Desert. Zeszyty Naukowe Uniwersytetu Jagiellonskiego, Prace Archeologiczne 35: 114-117.

1993 Evaluation of radiocarbon dates of organic samples from Uan Muhuggiag and Ti-n-Torha. In Krzyżaniak, L. and Kobusiewicz, M., eds., Environmental Change and Human Culture in the Nile Basin and Northern Africa Until the 2nd Millenium BC. Poznań, Poznań Archeological Museum: 43-47.

Pazdur, M. F., Awsiuk, R., Bluszcz, A., Goslar, T., Pazdur, A., Walanus, A. and Zastawny, A. 1985 Gliwice radiocarbon dates X. Radiocarbon 27(1): 52-73.

Pazdur, M. F., Awsiuk, R., Goslar, T., Michczyńska, D. J. and Pazdur, A. 1994 Radiocarbon chronology. In Ginter, B. and Kozlowski, J. K., eds., Predynastic Settlements Near Armant - Sites 21 and 21A. Heidelberg, Heidelberger Orientaler Verlag: 109-123.

Pazdur, M. F. and Michczyńska, D. J. 1989 Improvement of the procedure for probabilistic calibration of radiocarbon dates. In Long, A. and Kra, R. S., eds., Proceedings of the 13th International ${ }^{14} \mathrm{C}$ Conference. Radiocarbon 31(3): 824-832.

1993 Procedures for probabilistic calibration of radiocarbon dates with relevant specific examples. In Krzyżaniak, L. and Kobusiewicz, M., eds., Environmental Change and Human Culture in the Nile Basin and Northern Africa Until the 2nd Millenium BC. Poznań Archaeological Museum, Poznań: 471-481.

Prominska, E. 1984 The demography of the populations from Kadero (Central Sudan). In Krzyżaniak, L. and
Kobusiewicz, M., eds., Origin and Early Development of Food-Producing Cultures in North-Eastern Africa. Poznań, Polish Academy of Sciences and Poznań Archaeological Museum: 327-331.

Schild, R. and Wendorf, F. 1984 The earliest Holocene production of cereals in the Egyptian Sahara. In Krzyżaniak, L. and Kobusiewicz, M., eds., Origin and Early Development of Food-Producing Cultures in NorthEastern Africa. Poznań, Polish Academy of Sciences and Poznań Archaeological Museum: 373-379.

Wendorf, F., Close, A. E., Schild, R., Wasylikowa, K., Housley, R. A., Harlan, J. R. and Królik, H. 1992 Saharan exploitation of plants 8,000 years BP. Nature 359: 721-724.

Wendorf, F. and Schild, R., eds., 1980 Prehistory of the Eastern Sahara. New York, Academic Press.

Wendorf, F., Schild, R. and Close, A., eds., 1980 Loaves and Fishes: The Prehistory of Wadi Kubbaniya. Dallas, SMU Press.

1987 The Prehistory of Wadi Kubbaniya, Vol. 2. Stratigraphy, Paleoeconomy, and Environment. Dallas, Texas, Southern Methodist University Press.

Wildung, D. 1984 Terminal prehistory of the Nile Delta: theses. In Krzyżaniak, L. and Kobusiewicz, M., eds., Origin and Early Development of Food-Producing Cultures in North-Eastern Africa. Poznań, Polish Academy of Sciences and Poznań Archaeological Museum: 265-269. 